\title{
Solutions of Inviscid Burgers' and Equal Width Wave Equations by RDTM
}

\author{
Rajan Arora, Md. Junaid Siddiqui, and V. P. Singh
}

\begin{abstract}
In this paper, the Reduced Differential Transform method (RDTM) is used to find the numerical solution of the equal width wave (EW) equation and the exact analytical solution of the inviscid Burgers' equation with initial conditions. The method has been used successfully to investigate the motion of a single solitary wave that is governed by the $\mathrm{EW}$ equation. The solution is obtained in the form of convergent power series. The results obtained show that the error norms to the exact solutions are reasonably small and that the present method is easier and powerful than some other known techniques.
\end{abstract}

Index Terms - Single solitary wave, equal width wave (EW) equation, inviscid burgers' equation, reduced differential transform method (RDTM).

\section{INTRODUCTION}

It is commonly known that the equations of gas dynamics are the mathematical expressions of conservation laws which exist in engineering physics such as conservation of mass, conservation of momentum, conservation of energy etc. The inviscid equation of gas dynamics can be written in the conservation form which is a nonlinear partial differential equation. Partial differential equations have numerous applications in various fields such as fluid mechanics, physics, thermodynamics etc.

Most of these equations are nonlinear partial differential equations. A broad class of analytical solution

methods and numerical solution methods were applied to solve these partial differential equations by Morrison et al. [1], Gardner and Gardner [2], Smith [3], Courant and Friedrichs [4], Evans and Bulut [5], Wazwaz ([6] and [7]), He ([8] and [9]), Keskin [10], Peregrine [11], Bluman and Kumei [12], Whitham [13], Hunter and Keller [14], He and Moodie [15], Sharma and Radha [16], Sharma and Srinivasan [17] and Arora and Sharma [18] etc.wave equation.

The equal width wave equation, introduced by Morrison $e t$ al. [1], is of great importance since it is used as a model partial differential equation for the simulation of one dimensional wave propagation in nonlinear media with dispersion process.

In this Paper, we shall consider the Inviscid Burgers' equation

$$
u_{t}+\frac{\left(u^{2}\right) x}{2}=0
$$

with the initial condition $u(x, 0)=x$, and the Equal width wave equation (EW) ([1] and[2])

$$
u_{t}+u u_{x}-\mu u_{x x t}=0 \text {, }
$$

with the initial condition

$$
u(x, 0)=3 c \operatorname{sech}^{2}\left[p\left(x-x_{0}\right)\right],
$$

where $\mathrm{t}$ is time, $\mathrm{x}$ is the space coordinate, the dependent variable $u=u(x, t)$ is the wave amplitude, $\mu$ is the positive parameter, $c$ is the constant wave velocity, $p=\left(\frac{1}{4 \mu}\right)^{1 / 2}$ measures the width of the wave pulse and subscripts $t$ and $x$ denote partial differentiation, with the boundary condition $u \rightarrow 0$ as $x \rightarrow \pm \infty$.

The problem of the motion of single solitary wave governed by the EW equation (3) with the initial condition (4) has an exact solution of the form ([1] and [2])

$$
u(x, t)=3 c \operatorname{sech}^{2}\left[p\left(x-c t-x_{0}\right)\right] .
$$

\section{A. Analysis of the Method}

The basic definition of the Reduced Differential Transformation method [10] is as follows:

Definition I: If function $u(x, t)$ is analytic and $k$ times continuously differentiable with respect to time $t$ and space $x$ in the domain of interest, then let

$$
U_{k}(x)=\left(\frac{1}{k !}\right)\left(\frac{\partial^{k} u(x, t)}{\partial t^{k}}\right)_{t=0}
$$

where the t-dimensional spectrum function $U_{k}(x)$ is the transformed function of the original function $\mathrm{u}(x, t)$.

Definition II: The differential inverse transform of $U_{k}(x)$ is defined as follows

$$
u(x, t)=\sum_{k=0}^{\infty} U_{k}(x) t^{k} .
$$

Then combining these two equations we write

$$
u(x, t)=\sum_{k=0}^{\infty}\left(\frac{\partial^{k} u(x, t)}{\partial t^{k}}\right)_{t=0} t^{k}
$$

We write the inviscid Burgers' equation (1) in the standard operator form

$$
L(u(x, t))+N(u(x, t))=0,
$$

with initial condition $u(x, 0)=f(x)$, where $L(u(x, t))=\frac{\partial}{\partial t} u(x, t)$ is a linear term and $N(u(x, t))=\frac{\left(u^{2}\right) x}{2}$ is a nonlinear term.

According to RDTM, The transformed form of eq. (9) is

$$
(k+1) U_{k+1}(x)=-N\left(U_{k}(x)\right),
$$

where $N\left(U_{k}(x)\right)$ is the Reduced differential transformation of $N(u(x, t))$.

We shall also write the gas dynamics equation in standard Operator form

$$
L(u(x, t))-\mu R(u(x, t))+N(u(x, t))=0,
$$


with initial condition $\quad u(x, 0)=h(x)$,

where, $L(u)=u_{t}(x, t)$, is a linear term, $N(u(x, t))=\frac{\left(u^{2}\right)_{x}}{2}$ is a nonlinear term and $R=\frac{\partial^{3}}{\partial x^{2} \partial t}$ is a linear operator which has mixed partial derivatives. By the RDTM, we can construct the following iteration formula for equation

$$
\begin{aligned}
& (k+1) U_{k+1}(x)=-\sum_{r=0}^{k} U_{k-r}(x) \frac{\partial}{\partial x} U_{r}(x)+ \\
& \frac{\partial^{3}}{\partial x^{2} \partial t} \bar{u}_{k}(x, t), \quad(k=0,1,2, \ldots)
\end{aligned}
$$

Definition I implies that the initial approximation $U_{0}(x)$ is given by the initial condition, that is

$$
U_{0}(x)=u(x, 0) .
$$

Substituting $U_{0}(x)$ in the iteration formula (14), we get the values of $U_{k}(x)$. Then the differential inverse transformation of the set of values $\left[U_{k}(x)\right]_{k=0}^{n}$ gives approximation solution as

$$
\overline{\mathrm{u}}_{\mathrm{n}}(\mathrm{x}, \mathrm{t})=\sum_{\mathrm{k}=0}^{\mathrm{n}} \mathrm{U}_{\mathrm{k}}(\mathrm{x}) \mathrm{t}^{\mathrm{k}},
$$

where $\mathrm{n}$ is the order of approximation solution. Hence the exact solution of the problem is given by

$$
u(x, t)=\lim _{n \rightarrow \infty} \bar{u}_{n}(x, t) .
$$

\section{B. Applications}

Problem 1: We consider the Inviscid Burgers' equation

$$
u_{t}+\frac{\left(u^{2}\right) x}{2}=0
$$

with the initial condition $u(x, 0)=x$,

where $u=u(x, t)$ is a function of the variables $\mathrm{x}$ and $\mathrm{t}$. The exact solution of this problem is $(x, t)=\frac{x}{1+t}$.

By RDTM, The transformed form of eq. (18) is

$$
(k+1) U_{k+1}(x)=-N\left(U_{k}(x)\right),
$$

where $N\left(U_{k}(x)\right)$ is the Reduced differential transformation of $\frac{\left(\mathrm{u}^{2}\right)_{\mathrm{x}}}{2}$.

Using the initial condition (19), we get the initial approximation as

$$
U_{0}(x)=x .
$$

Now substituting (21) into (20), we get the successive values of $U_{k}(x)$ as

$U_{1}(x)=-x, U_{2}(x)=x, \quad U_{3}(x)=-x, \quad U_{4}(x)=$

$x, U_{5}(x)=-x, \ldots$

The Differential inverse transform of $\mathrm{U}_{\mathrm{k}}(\mathrm{x})$ gives

$$
\begin{aligned}
u(x, t)=\sum_{k=0}^{\infty} U_{k}(x) t^{k} & =x\left[1-t+t^{2}-t^{3}+\ldots\right] \\
& =\frac{x}{1+t},
\end{aligned}
$$

which is the exact solution of (18) with the initial condition (19).

Problem 2: For the sake of convenience and without any loss of generality, we assume $\mu=1, c=0.1$, and $x_{0}=$ 10 in the EW wave equation (3)-(4) to obtain

$$
u_{t}+u u_{x}-u_{x x t}=0 \text {, }
$$

with initial condition

$$
u(x, 0)=0.3 \operatorname{sech}^{2}(0.5 x-5) .
$$

This problem has an exact solution of the form ([1] and [2])

$$
u(x, t)=0.3 \operatorname{sech}^{2}(0.5 x-0.05 t-5) .
$$

Taking Reduced differential transformation of (23), we get $(k+1) U_{k+1}(x)=-\sum_{r=0}^{k} U_{k-r}(x) \frac{\partial}{\partial x} U_{r}(x)+$ $\frac{\partial^{3}}{\partial x^{2} \partial t} \bar{u}_{k}(x, t), \quad(k=0,1,2, \ldots)$,

where the function $U_{k}(x)$ is the transformed function of the original function $\mathrm{u}(\mathrm{x}, \mathrm{t})$ and $\bar{u}_{n}(x, t)=\sum_{k=0}^{n} U_{k}(x) t^{k}$ is the $\mathrm{n}^{\text {th }}$ order approximation solution of (23). From the Initial condition (24), Initial approximation is given by

$$
U_{0}(x)=0.3 \operatorname{sech}^{2}(0.5 x-5) .
$$

Substituting (27) into (26), we obtain the successive values of $U_{k}(x)$ as

$$
U_{1}(x)=0.09 \operatorname{sech}^{4}(0.5 x-5) \tanh (0.5 x-5),
$$

$U_{2}(x)=$

$-0.04625 \operatorname{sech}^{8}(0.5 x-5)+0.0395 \operatorname{sech}^{6}(0.5 x-5)-$

$0.3375 \operatorname{sech}^{6}(0.5 x-5) \tanh (0.5 x-5)+$

$0.18 \operatorname{sech}^{4}(0.5 x-5) \tanh (0.5 x-5)$,

and so on.

Then, the differential inverse transformation of the set of values $\left[U_{k}(x)\right]_{k=0}^{2}$ gives the second order approximation solution as

$$
\bar{u}_{2}(x, t)=\sum_{k=0}^{2} U_{k}(x) t^{k}=U_{0}+U_{1}(x) t+U_{2}(x) t^{2}
$$

$$
\begin{aligned}
=0.3 \operatorname{sech}^{2}(0.5 x & -5) \\
& +0.09 t \operatorname{sech}^{4}(0.5 x-5) \tanh (0.5 x-5) \\
& -0.04625 t^{2} \operatorname{sech}^{8}(0.5 x-5) \\
& -0.3375 t^{2} \operatorname{sech}^{6}(0.5 x-5) \tanh (0.5 x \\
& -5) \\
& +0.18 t^{2} \operatorname{sech}^{4}(0.5 x-5) \tanh (0.5 x \\
& -5) .
\end{aligned}
$$

Therefore the exact solution of (23) with the initial condition (24) is given by

$$
u(x, t)=\lim _{n \rightarrow \infty} \bar{u}_{n}(x, t) .
$$

This solution is convergent to the exact solution

$$
u(x, t)=0.3 \operatorname{sech}^{2}(0.5 x-0.05 t-5) .
$$

The comparison of the present numerical solutions with the exact solutions of the EW equation is made in the following table:

\begin{tabular}{|c|c|ccc|}
\hline $\mathrm{t}$ & $\mathrm{x}$ & Approx. sol. RDTM & Exact solution & Absolute Error \\
\hline \multirow{2}{*}{0.003} & -1 & $0.2004137028 \mathrm{E}-4$ & $0.2003536018 \mathrm{E}-4$ & $0.60101 \mathrm{E}-8$ \\
& 0 & $0.5547496033 \mathrm{E}-4$ & $0.5445863073 \mathrm{E}-4$ & $0.163296 \mathrm{E}-7$ \\
& 1 & $0.14805511978 \mathrm{E}-3$ & $0.1480108208 \mathrm{E}-3$ & $0.44299 \mathrm{E}-7$ \\
\hline \multirow{2}{*}{0.001} & -1 & $0.2004137109 \mathrm{E}-4$ & $0.2003936752 \mathrm{E}-4$ & $0.200357 \mathrm{E}-8$ \\
& 0 & $0.1480551197 \mathrm{E}-3$ & $0.1480404186 \mathrm{E}-3$ & $0.147012 \mathrm{E}-7$ \\
\hline \multirow{2}{*}{0.01} & 1 & $0.2004136740 \mathrm{E}-4$ & $0.2002134081 \mathrm{E}-4$ & $0.200266 \mathrm{E}-7$ \\
& -1 & $0.5447493901 \mathrm{E}-4$ & $0.5442052648 \mathrm{E}-4$ & $0.544125 \mathrm{E}-7$ \\
& 0 & $0.1480549962 \mathrm{E}-3$ & $0.1479072750 \mathrm{E}-3$ & $0.147721 \mathrm{E}-6$ \\
\hline
\end{tabular}




\section{CONCLUSION}

The Reduced Differential Transform Method (RDTM) has been used to obtain numerical solutions of the inviscid Burgers' equation and the equal width wave (EW) equation with initial conditions. The efficiency of the RDTM was tested on these equations with initial conditions. The method provides an analytical approximation, some time an exact solution, in the form of convergent power series. The comparison of the numerical solution obtained by this method to the exact solution in Table 1 shows that the numerical results are in very good agreement with the exact solution. The results are also depicted in Figure 1 and Fig. 2. Authors submitting a manuscript do so on the understanding that if the manuscript is accepted for publication, copyright for the article, including the right to reproduce the article in all forms and media, shall be assigned exclusively to the Publisher.

\section{ACKNOWLEDGEMENT}

Research fundings from the Department of Science and Technology (DST), Government of India, New Delhi, vide sanction order no. SR/FTP/MS-12/2008 and from the CSIR, New Delhi vide reference number 09/143 (0765)/2010EMR-I are gratefully acknowledged.

\section{REFERENCES}

[1] P. J. Morrison, J. D. Meiss, and J. R. Cary, "Scattering of RLW Solitary Waves," Physica D, Non- linear Phenomena 1984, vol. 11, pp. 324-336.

[2] L. R. T. Gardner and G. A. Gardner, "Solitary waves of the equal width wave equation," Journal of Computational Physics, 1992, vol. 101, pp. $218-223$.
[3] C. D. Smith, "Numerical Solution of Partial Differential Equations," Claredon Press, Oxford, 1978.

[4] R. Courant and K.O. Friedrichs, "Supersonic flows and shock waves," Springer Verlag, New York, 1976.

[5] D. J. Evans and H. Bulut, "A new approach to the gas dynamics equation, an application of the decomposition method," International Journal of Computer Mathematics 2002, vol. 79, no. 7, 817822.

[6] A. M. Wazwaz, "Partial Differential equations: methods and applications," The Netherlands Balkema Publishers, 2002.

[7] A. M. Wazwaz, "A Sine-Cosine method for handling Nonlinear Wave Equations," Mathematical and Computer modelling 2004, vol. 40, pp. 499-508.

[8] J. H. He, "Variational iteration method-a kind of non-linear analytical technique: Some examples," International Journal of Non-Linear Mechanics 1999, vol. 34, no. 4, pp. 699-708.

[9] J. H. He, "Homotopy Perturbation method: A new nonlinear technique," Applied Mathematics and computation 2003, vol.135, pp. 73-79.

[10] Y. Keskin and G. Oturanc, "Reduced Differential Transform Method for Partial Differential equations," International Journal of Nonlinear Sciences and Numerical Simulation, 2009, vol. 10, no. 6, pp. 741-749.

[11] D. H. Peregrine, "Calculations of the development of an undular bore," J. Fluid Mech 1966, vol. 25, pp. 321-330.

[12] G. W. Bluman and S. Kumei. Symmetries and Differential Equations, Springer, New York 1989.

[13] G. B. Whitham. Linear and NonLinear Waves, Wiley-Interscience, New York 1974.

[14] J. K. Hunter and J. Keller, "Weakly nonlinear high frequency waves," Comm. Pure Appl. Math. 1983, vol. 36, pp. 547-569.

[15] Y. He and T. B. Moodie, "Two-wave interactions for weakly nonlinear hyperbolic waves," Stud. Appl. Math. 1993, vol. 88, pp. 241-267.

[16] V. D. Sharma and Ch. Radha, "Similarity solutions for converging shock in a relaxing gas," Int. J. Engg. Science 1995, vol. 33, pp. 535-553.

[17] V. D. Sharma and Gopala Krishna Srinivasan, "Wave interaction in a non-equilibrium gas flow," Int. J. Non-linear Mechanics 2005, vol. 40, pp. 1031-1040.

[18] Rajan Arora and V.D. Sharma, "Convergence of Strong Shock in a Van der Waals gas," SIAM J. Applied Mathematics 2006, vol. 66, pp. 1825-1837. 